\title{
A General Direct Arc Interpolation Method
}

\author{
Zefan Cai \\ Shunde Polytechnic \\ Foshan, China \\ South China University of Technology \\ Guangzhou, China \\ Email: 12266423@qq.com
}

\author{
Daoping Huang \\ Automation College \\ South China University of Technology \\ Guangzhou, China \\ audhuang@scut.edu.cn
}

\begin{abstract}
Circular interpolation methods are mostly introduced based on CNC machines, and point by point judgment methods are used to determine the end of the arcs, but these methods are less common and less efficient. This paper introduces a universal direct arc interpolation method. Using this method, all the points on an arc can be conveniently calculated in a time, without continuous judgment in the whole fitting procedure. This method is highly efficient and has no need to worry if the arc can reach the right end. The circular interpolation control method can be applied to CNC systems, such as CNC machine, multi-dof manipulator, etc..
\end{abstract}

Keywords-arc; direct interpolation; fitting; normal vector; numerical control technology

\section{INTRODUCTION}

In the numerical control system, especially in $\mathrm{CNC}$ machines system, the interpolation method usually adopts point by point judgment methods.[1-5] In these methods, the circle center is generally considered as the origin of a coordinate system, and the interpolation is processed according to the start point and the end point. [1,2] This method is suitable for the application of CNC machine systems, but not suitable for more general numerical control system, such as multi-dof robot. More general method is to obtain the arc crossing all the three setting points. Reference [6] does not use point by point interpolation method, but only the two dimension situations are discussed, and the arc in different quadrant is obtained independently. Reference [7] and [8] used the sampling circular interpolation method, but it still depends on different quadrants. The interpolation equation of each quadrant is different, and the equation in clockwise and anticlockwise situations is also different, which is inconvenient for programming.

In this paper, a simple direct circular interpolation method is introduced, which does not depend on quadrants All the points can be obtained in a time if three points are known which aren't in a line. The circular interpolation problem based on three points in space is to obtain the arc which will cross all the three setting points (x1, y1, z1), $(x 2, y 2, z 2)$ and $(x 3, y 3, z 3)$. From the mathematical knowledge, it can be known that as long as three points are non collinear and concurrent, in other words, the three points can be the three vertices of a triangle, that the circu mcircle of the three points can be determined. After the circumcircle is obtained, the arc is easy to obtain. [9]
The method to obtain the circumcircle is to calculate the center and radius first, and then calculate each point on the circle.

\section{CIRCLE CENTER AND RADIUS}

If the radius of the circle determined by the three points is $r$ and the center is $(x, y, z)$, the relation between the radium and center is as follow:

$$
\begin{aligned}
& r^{2}=\left(x_{1}-x\right)^{2}+\left(y_{1}-y\right)^{2}+\left(z_{1}-z\right)^{2} . \\
& r^{2}=\left(x_{2}-x\right)^{2}+\left(y_{2}-y\right)^{2}+\left(z_{2}-z\right)^{2} . \\
& r^{2}=\left(x_{3}-x\right)^{2}+\left(y_{3}-y\right)^{2}+\left(z_{3}-z\right)^{2} .
\end{aligned}
$$

According to (1) and (2), it can be got

$$
\begin{aligned}
& 2\left(x_{2}-x_{1}\right)+2\left(y_{2}-y_{1}\right)+2\left(z_{2}-z_{1}\right) \\
& +x_{1}^{2}+y_{1}^{2}+z_{1}^{2}-x_{2}^{2}-y_{2}^{2}-z_{2}^{2}=0
\end{aligned}
$$

Equation (4) can be rewritten as follow

$$
A_{2} x+B_{2} y+C_{2} z+D_{2}=0 \text {. }
$$

According to (1) and (3), it can be got

$$
2\left(x_{3}-x_{1}\right)+2\left(y_{3}-y_{1}\right)+2\left(z_{3}-z_{1}\right)
$$$$
+x_{1}^{2}+y_{1}^{2}+z_{1}^{2}-x_{3}^{2}-y_{3}^{2}-z_{3}^{2}=0
$$

Equation (6) can be rewritten as follow

$$
A_{3} x+B_{3} y+C_{3} z+D_{3}=0 \text {. }
$$

Any three points (x1, y1, z1), (x2, y2, z2) and $(x 3, y 3, z 3)$, which aren't in the same line, can determine a plane, if $(\mathrm{x}, \mathrm{y}, \mathrm{z})$ is a random point of the plane, then the plane equation is [9]

$$
\left|\begin{array}{llll}
x & y & z & 1 \\
x_{1} & y_{1} & z_{1} & 1 \\
x_{2} & y_{2} & z_{2} & 1 \\
x_{3} & y_{3} & z_{3} & 1
\end{array}\right|=0
$$

Equation (8) can be expressed as

$$
A_{1} x+B_{1} y+C_{1} z+D_{1}=0
$$

where

$$
\begin{aligned}
& A_{1}=y_{1} \cdot z_{2}-y_{1} \cdot z_{3}-z_{1} \cdot y_{2}+z_{1} \cdot y_{3}+y_{2} \cdot z_{3}-y_{3} \cdot z_{2}, \\
& B_{1}=-x_{1} \cdot z_{2}+x_{1} \cdot z_{3}+z_{1} \cdot x_{2}-z_{1} \cdot x_{3}-x_{2} \cdot z_{3}+x_{3} \cdot z_{2}, \\
& C_{1}=x_{1} \cdot y_{2}-x_{1} \cdot y_{3}-y_{1} \cdot x_{2}+y_{1} \cdot x_{3}+x_{2} \cdot y_{3}-x_{3} \cdot y_{2}, \\
& D_{1}=-x_{1} \cdot y_{2} \cdot z_{3}+x_{1} \cdot y_{3} \cdot z_{2}+x_{2} \cdot y_{1} \cdot z_{3} \\
& -x_{3} \cdot y_{1} \cdot z_{2}-x_{2} \cdot y_{3} \cdot z_{1}+x_{3} \cdot y_{2} \cdot z_{1}
\end{aligned}
$$


Equation (5), (7) and (9) can be expressed as equation set in matrix manner which is

$$
\left[\begin{array}{lll}
A_{1} & B_{1} & C_{1} \\
A_{2} & B_{2} & C_{2} \\
A_{3} & B_{3} & C_{3}
\end{array}\right]\left[\begin{array}{l}
x \\
y \\
z
\end{array}\right]+\left[\begin{array}{c}
D_{1} \\
D_{2} \\
D_{3}
\end{array}\right]=\mathbf{0}
$$

The solution of (10) is

$$
\left[\begin{array}{l}
x_{0} \\
y_{0} \\
z_{0}
\end{array}\right]=\left[\begin{array}{lll}
A_{1} & B_{1} & C_{1} \\
A_{2} & B_{2} & C_{2} \\
A_{3} & B_{3} & C_{3}
\end{array}\right]^{-1}\left[\begin{array}{c}
D_{1} \\
D_{2} \\
D_{3}
\end{array}\right] .
$$

$(\mathrm{x} 0, \mathrm{y} 0, \mathrm{z} 0)$ is the center coordinate of the circle.

The radius $r$ is

$$
r=\sqrt{\left(x_{1}-x_{0}\right)^{2}+\left(y_{1}-y_{0}\right)^{2}+\left(z_{1}-z_{0}\right)^{2}} \text {. }
$$

For example, if $(\mathrm{x} 1, \mathrm{y} 1, \mathrm{z} 1)=(-1,2,3),(\mathrm{x} 2, \mathrm{y} 2, \mathrm{z} 2)=$ $(2,4,4)$ and $(\mathrm{x} 3, \mathrm{y} 3, \mathrm{z} 3)=(3,-6,6)$, then $(\mathrm{x} 0, \mathrm{y} 0, \mathrm{z} 0)=$ $(2.6325,-0.9759,5.0542)$ and $\mathrm{r}=5.1255$.

\section{CiRCLE FITTING}

The normal vector $\mathrm{n}$ of the plane can be obtained by the plane equation. The vector from $(\mathrm{x} 0, \mathrm{y} 0, \mathrm{z} 0)$ to $(\mathrm{x} 1$, $\mathrm{y} 1, \mathrm{z} 1)$ can be denoted as V1. Then the vertical vector V2 of vector V1 can be obtained with $\mathrm{n}$ and V1. [10] If ( $\mathrm{x}, \mathrm{y}, \mathrm{z})$ is a random point of the circle which needs to be fitted, the vector from $(\mathrm{x} 0, \mathrm{y} 0, \mathrm{z} 0)$ to $(\mathrm{x}, \mathrm{y}, \mathrm{z})$ is $\mathrm{V}$, and the angle between $\mathrm{V}$ and $\mathrm{V} 1$ is $\theta$, then $(\mathrm{x}, \mathrm{y}, \mathrm{z})$ is

$$
\left[\begin{array}{l}
x \\
y \\
z
\end{array}\right]=\left[\begin{array}{l}
x_{0} \\
y_{0} \\
z_{0}
\end{array}\right]+\cos (\theta) V_{1}+\sin (\theta) V_{2}
$$

If $\theta$ increases from $0^{\circ}$ to $360^{\circ}$ in a certain increment, such as $0.1^{\circ}$, then a series of points will be obtained. The circle will be fitted by connecting all these points in order. The circle in Fig. 1 is fitted in this way.

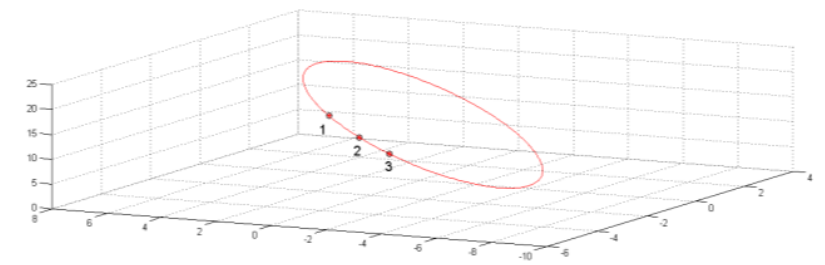

Figure 1. A circle fitted by three points

\section{ARC FITTING}

When a circle needs to be fitting, it's no need to care about which are the starting point and end point. A circle can be fitted with (13) as long as the variation of $\theta$ is $360^{\circ}$ out the starting point and end point. $\mathrm{F}$ want to obtain an arc crossing the three points in Fig. 1, there are three scenarios $-1 \rightarrow 2 \rightarrow 3,1 \rightarrow 3 \rightarrow 2$ and $2 \rightarrow 1 \rightarrow 3$.

For a specific interpolation problem, the order of the three points is clear, put the first point as a starting point, the last point as the end point. In the program, the choice of the starting point is easy, but it is difficult to choose the end point. Fitting accuracy depends on the choice of the end point. Furthermore, if the choice is wrong, it is possibly unable to finish the fitting. There are several methods to determine the end point. Reference [2] introduces three methods to judge the end point. They are distance judging method, angle judging method and matching judging method. All the three methods are point by point judging method. In these methods, the difference between the current point and the end point is calculated continuously during the whole fitting process. It can achieve the final judgment based on the three methods, but the efficiency is very low. Reference [3] studies five methods to determine the end point, respectively, the sign of the difference between the current interpolation point and the end point, distance judging method (a), distance judging method (b), coordinate distance judging method, combination of the distance judging method and the change of sign. Although there are differences in the various methods, but they are still point by point methods.

This paper introduces a direct arc interpolation method. Using this method, all the points on an arc can be conveniently calculated in a time, without continuous judgment in the whole fitting process. This method is highly efficient and has no need to worry if the arc interpolation can finish. In order to facilitate the problem, take the unit circle as an example. One of the dimensions is set to 0 . The starting point is $\mathrm{A}=(\mathrm{x} 1, \mathrm{y} 1, \mathrm{z} 1)=(0,1,0)$, the middle point is $\mathrm{B}=(\mathrm{x} 2, \mathrm{y} 2, \mathrm{z} 2)$, the ending point is $\mathrm{C}=$ $(\mathrm{x} 3, \mathrm{y} 3, \mathrm{z} 3)$ and the circle center is $\mathrm{O}=(\mathrm{x} 0, \mathrm{y} 0, \mathrm{z} 0)=(0$, $0,0)$. According to (13), this starting point is on Y-axis, which are shown in Fig. 2 and Fig. 3.

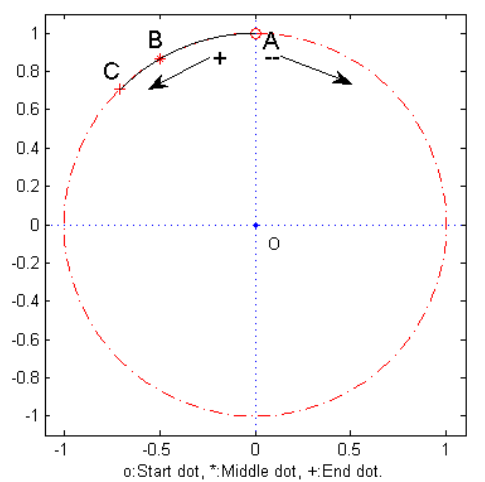

Figure 2. Circle crossing three points and sign of angle (1)

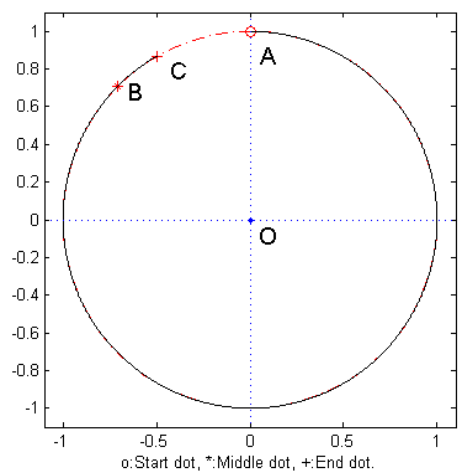

Figure 3. Circle crossing three points and sign of angle (2)

As shown in Fig. 2, the angle between the vector OA and the Y-axis is $0^{\circ}$, the angle between the vector $\mathrm{OB}$ and 
OA is $\theta_{1}$,the angle between the vector OA and OC is $\theta_{2}$, the sign of the anticlockwise angle is + , while the sign of the clockwise angle is -.

$$
\begin{aligned}
& \theta_{1} \text { is } \\
& \theta_{1}=\arccos \left(\begin{array}{l}
\left(x_{1} x_{2}+y_{1} y_{2}+z_{1} z_{2}\right)^{\prime} \\
\left(\sqrt{x_{1}^{2}+y_{1}^{2}+z_{1}^{2}} \sqrt{x_{2}^{2}+y_{2}^{2}+z_{2}^{2}}\right)
\end{array}\right) . \\
& \theta_{2} \text { is } \\
& \theta_{2}=\arccos \left(\begin{array}{l}
\left(x_{1} x_{3}+y_{1} y_{3}+z_{1} z_{3}\right) / \\
\left(\sqrt{x_{1}^{2}+y_{1}^{2}+z_{1}^{2}} \sqrt{x_{3}^{2}+y_{3}^{2}+z_{3}^{2}}\right)
\end{array}\right)
\end{aligned}
$$

All the position relations of the point $\mathrm{B}$ and $\mathrm{C}$ are listed in Table 1.

TABLE I. The Position RELATION OF MidDLE PoINT AND END POINT AND INTERPOLATION RESULT

\begin{tabular}{|c|c|c|}
\hline Position of B & Position of C & Interpolation Result \\
\hline 1st quadrant; Front & 1st quadrant; Back & Correct \\
\hline 1st quadrant; Back & 1st quadrant; Front & Correct \\
\hline 1 st quadrant & 2nd quadrant & Correct \\
\hline 1st quadrant & 3rd quadrant & Correct \\
\hline 1st quadrant & 4th quadrant & Correct \\
\hline 2nd quadrant & 1 st quadrant & Correct \\
\hline 2nd quadrant; Front & 2nd quadrant; Back & Correct \\
\hline 2nd quadrant; Back & 2nd quadrant; Front & Correct \\
\hline 2nd quadrant & 3rd quadrant & Correct \\
\hline 2nd quadrant & 4th quadrant & Correct \\
\hline 3rd quadrant & 1st quadrant & Correct \\
\hline 3rd quadrant & 2nd quadrant & Correct \\
\hline 3rd quadrant; Front & 3rd quadrant; Back & Correct \\
\hline 3rd quadrant; Back & 3rd quadrant; Front & Correct \\
\hline 3rd quadrant & 4th quadrant & Correct \\
\hline 4th quadrant & 1st quadrant & Correct \\
\hline 4th quadrant & 2nd quadrant & Correct \\
\hline 4th quadrant & 3rd quadrant & Correct \\
\hline 4th quadrant; Front & 4th quadrant; Back & Correct \\
\hline 4th quadrant; Back & 4th quadrant; Front & Correct \\
\hline When the mide
\end{tabular}

When the middle point and the ending point are in the same quadrant, from the starting point, in a anticlockwise direction, the point reached first is defined as the front point and another is defined as the back point. In order to facilitate comparison, $\theta_{1}$ and $\theta_{2}$ are always set to positive values. When their values are negative, add $360^{\circ}$ to the values and make them positive.

As shown in Fig. 2 and Fig. 3, when the positions of B and $\mathrm{C}$ are different, the interpolating arc is different. In Fig. 2 , the interpolating arc is from $\mathrm{A}$ to $\mathrm{C}$ in anticlockwise direction, while in Fig. 3, the arc is from $\mathrm{A}$ to $\mathrm{C}$ in clockwise direction.

In interpolation, the starting angle is defined as $\theta_{s}$, its value is

$$
\theta_{s}=\left\{\begin{array}{c}
0^{\circ}, \theta_{2}>\theta_{1} \\
360^{\circ}, \theta_{2}<\theta_{1} .
\end{array}\right.
$$

the end angle is defined as $\theta_{e}$, its value is $\theta_{e}=\theta_{2}$
$\mathrm{M}$ is a random point in the arc. The angle between vector $\mathrm{OM}$ and $\mathrm{OA}$ is $\theta$. When $\theta$ grows from $\theta_{s}$ to $\theta_{e}$ in a certain increment, such as $0.1^{\circ}$, a series of $\mathrm{M}$ will be obtained with (13). Then the required arc will be fitted by connecting adjacent points with short lines in order.

\section{SIMULATION VERIFICATION AND CONCLUSION}

The method is verified by simulation in MATLAB. The simulation result shows that all the 20 cases listed in Table 1 can be correctly interpolated. Six cases are shown in Fig. 4 to Fig. 9, where "o" represents the starting point, "*" represents the middle point, "+" represents the end point, and the solid line represents the actual fitting arc. From Fig. 4 to Fig. 9, it can be seen that all the fitting arc starts from the starting points, then cross the middle point, and at last reach the end point.

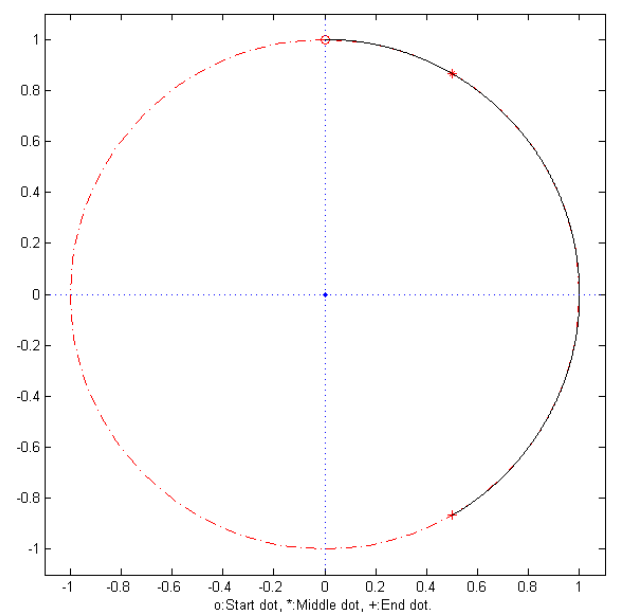

Figure 4. Arc Interpolation (a)

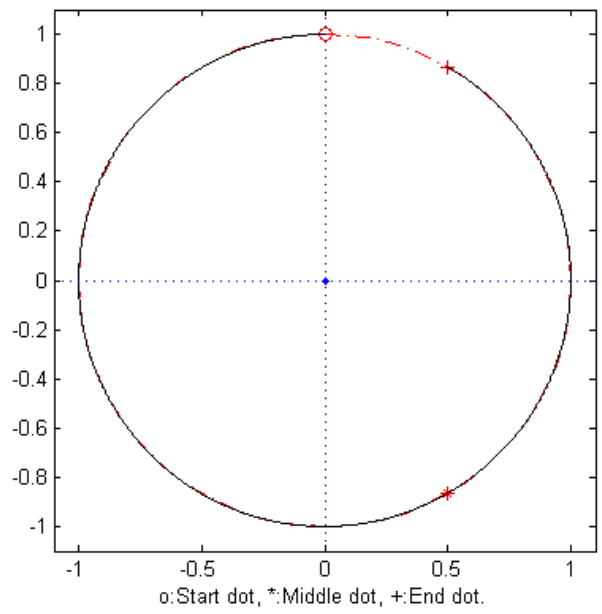

Arc Interpolation (b) 


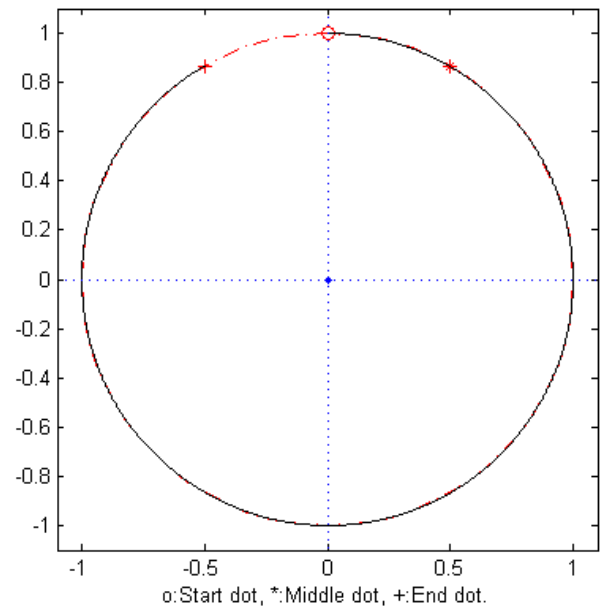

Figure 5. Arc Interpolation (c)

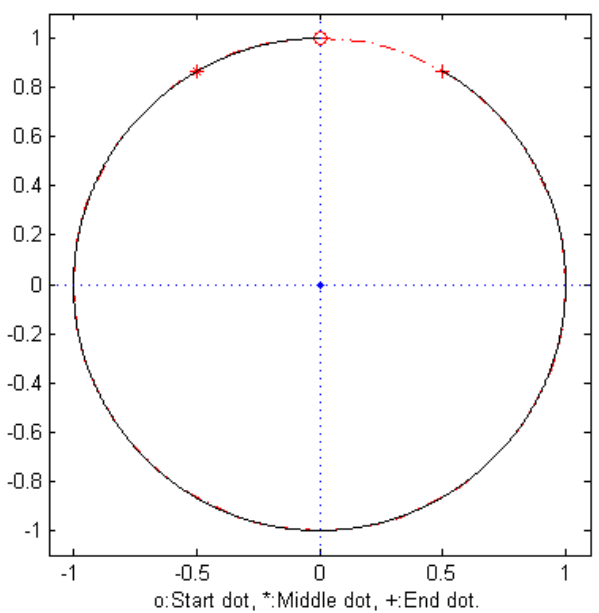

Figure 6. Arc Interpolation (d)

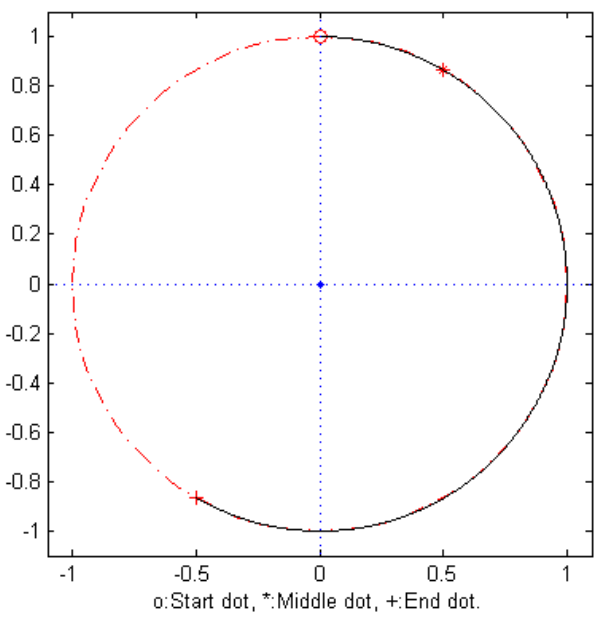

Figure 7. Arc Interpolation (e)

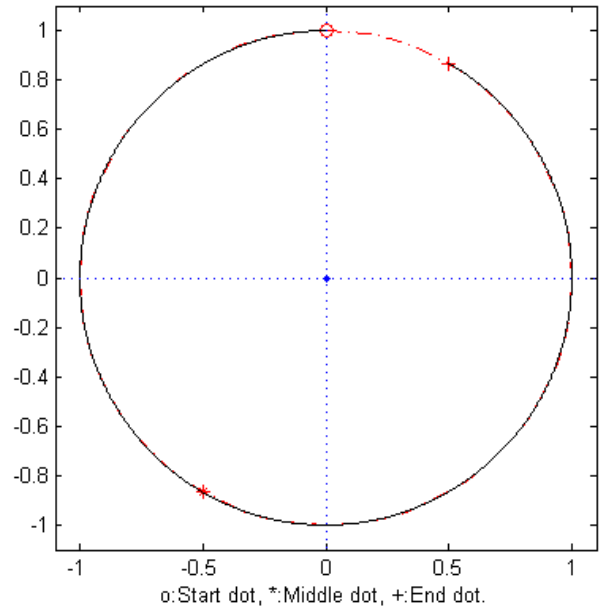

Figure 8. Arc Interpolation (f)

Through theoretical analysis and simulation verification, it is proved that the circular interpolation method introduced in this paper is effective. It avoids judging the end point continuously in the whole interpolating procedure, and improves the fitting speed. This method can be applied to the circular interpolation in the numerical control system, especially effective in the embedded system.

\section{REFERENCE}

[1] Li Enlin. Principle and application of numerical control technology, National Defence Industry Press, 2006.

[2] Lin Benjie, Shi Chuan, Zhang Hui, Ye Peiqing. "Study on Endpoint Discrimination Methods of Circular Interpolation", Manufacturing Technology \& Machine Tool, vol. 4, 2007, pp. 8689.

[3] Wang Min. "Research on Comparation between 2 Algorithms of Arc Interpolation \& 5 Methods of End-point Discrimination", Modular Machine Tool \& Automatic Manufacturing Technique, vol. 3, 1996, pp. 5-10.

[4] Chen Fei. "Discussion on End-point Judgment of Circular Interpolation of Point by Point Comparison Method", Equipment Manut acturing Technology, vol. 11, 2014, pp. 177, 178, 188.

[5] Wang Wenxi. "Research on End-point Judgment of Point-by-point Interpolation", Journal of Qingdao University, vol. 12(1), 1999, pp. 86-90.

[6] Shuai Qi. "Circular and Elliptical Interpolation Algorithm Based on Least Deviation of the Interpolation Function", Science \& Technology Vision, 2014,pp. 237, 267.

[7] Zhou Hui. "The new calculation way on circular interpolation of the data sampling kind method", Modular Machine Tool \& Aut omatic Manufacturing Technique, vol. 2, 2004, pp. 38, 39, 42.

[8] Li Li, Feng Zhiyong. "Improvement \& Realization of the Circle Interpolation Algorithm in CNC System", Modular Machine Tool \& Aut omatic Manufacturing Technique, vol. 11, 2014, pp. 177, $178,188$.

[9] Department of Applied Mathematics of Tongii University. Linear algebra, Higher Education Press, 2003.

[10] Mei Li. "Plane equation of two intersecting lines generated in space", Journal of Nanyang Teachers College, vol. 12(12), 2013, pp. 16-19. 